\title{
Is the Free Vacuum Energy Infinite?
}

\author{
H. Razmi and S. M. Shirazi \\ Department of Physics, The University of Qom, Qom 3716146611, Iran \\ Correspondence should be addressed to H. Razmi; razmi@qom.ac.ir \\ Received 12 February 2015; Revised 14 April 2015; Accepted 16 April 2015 \\ Academic Editor: Chao-Qiang Geng
}

Copyright (C) 2015 H. Razmi and S. M. Shirazi. This is an open access article distributed under the Creative Commons Attribution License, which permits unrestricted use, distribution, and reproduction in any medium, provided the original work is properly cited. The publication of this article was funded by SCOAP ${ }^{3}$.

Considering the fundamental cutoff applied by the uncertainty relations' limit on virtual particles' frequency in the quantum vacuum, it is shown that the vacuum energy density is proportional to the inverse of the fourth power of the dimensional distance of the space under consideration and thus the corresponding vacuum energy automatically regularized to zero value for an infinitely large free space. This can be used in regularizing a number of unwanted infinities that happen in the Casimir effect, the cosmological constant problem, and so on without using already known mathematical (not so reasonable) techniques and tricks.

\section{Introduction}

In the standard quantum field theory, not only does the vacuum (zero-point) energy have an absolute infinite value, but also all the real excited states have such an irregular value; this is because these energies correspond to the zero-point energy of an infinite number of harmonic oscillators $(W=$ $\left.(1 / 2) \sum_{k, \sigma} \hbar \omega_{k} \rightarrow \infty\right)$. We usually get rid of this irregularity via simple technique of normal ordering by considering the energy difference relative to the vacuum state [1-6]; but, of course, there are some important situations where one deals directly with the absolute vacuum energy as in the cosmological constant problem [7] or in the regularization of the vacuum energy in the Casimir effect [8]. After a half century of knowing and "living" with the vacuum energy, there are only some mathematical techniques and approaches in regularizing its infinite value without paying enough conceptual attention to the "content" of the quantum vacuum "structure." In this paper, considering the fundamental assumption that the vacuum energy originates from the "motion" of virtual particles in the quantum vacuum, it is shown that the free vacuum energy can be regularized based on the uncertainty relations' limit on these particles' frequency. Indeed, the free vacuum (or any infinitely large vacuum space) energy is automatically regularized to zero value without using any presupposition (e.g., normal ordering) usually used in getting rid of the infinity of the quantum vacuum energy (the vacuum catastrophe).

\section{The Quantum Vacuum, Virtual Particles, and the Uncertainty Relations}

The quantum vacuum is not really empty. It is filled with virtual particles which are in a continuous state of fluctuation. Virtual particle-antiparticle pairs are created from vacuum and annihilated back to it. These virtual particles exist for a time dictated by Heisenberg uncertainty relation. Based on the uncertainty relations, for any virtual particle, there is a limit on the timescale of "being" created from the vacuum fluctuations and then annihilated back to vacuum (its "lifetime"); thus, there should be a limit on the frequency of the virtual particles whose total energies are considered as the vacuum energy. In quantum (field) theory, it is well known that the reason for naming the quantum vacuum particles as virtual particles is that although they are in "existence" and can have observable effects (e.g., the Casimir effect, spontaneous emission, and Lamb shift), they cannot be directly detected (i.e., they are unobservable). For these unobservable (virtual) particles, the energy and lifetime values are constrained due to the uncertainty relation and can take, at most, the minimum values of uncertainties for real particles. This can be written as the following relation:

$$
\left.(E \tau)\right|_{\max .} ^{\text {virtual }} \approx \alpha \frac{\hbar}{2},
$$

where $\alpha$ is a constant which cannot have a value much more than 1 to guarantee that we are dealing with virtual particles 
instead of real ones. As we know, the uncertainties in energy and lifetime of real (detectable) particles satisfy the relation

$$
\Delta E \Delta \tau \geq \frac{\hbar}{2}
$$

\section{The Vacuum Energy Density of Infinitely Large Free Spaces}

Although attribution of physical parameters and quantities to the virtual particles, the same as what we know for the real particles, is not a completely known and proved fact, the main reason of irregularity/infinity of the vacuum energy in QFT is because of attribution of the frequency $\omega$ to the virtual particles and summing on the infinite modes for them. Also, attributing "distance" to virtual particles is a known fact; in the Casimir effect, we talk about the confinement of virtual particles in a finite distance between the two plates and the Casimir force depends on this distance. The only known point about the "attendance" of the virtual photons in a finite distance in QFT is that these intermediate particles (as in the Feynman diagrams) have nonzero masses with finite range of "action"; this makes them have a velocity of $v<c$, where we will consider it in our calculation.

For a free space of dimensional length $D$, using the relations $E=\hbar \omega, \tau \leq D / c$, and (1), the frequency of virtual particles should satisfy

$$
\left.\omega\right|^{\text {virtual }} \leq \frac{\alpha c}{2 D} .
$$

Considering this limit on $\omega$ and the following well-known relation for the vacuum energy density corresponding to an infinitely large space

$$
\frac{E}{V}=\frac{1}{2 V} \sum_{k} \hbar \omega_{k} \longrightarrow 2 \pi \hbar c \int k^{3} d k
$$

it is found that

$$
\frac{E}{V}=2 \pi \hbar c \int_{0}^{\alpha / 2 D} k^{3} d k=\frac{\pi \hbar c \alpha^{4}}{8 D^{4}} .
$$

For an infinitely large free space, the vacuum energy is zero; it is automatically regularized as in the following:

$$
\begin{aligned}
E_{\text {free }} & =\left(\frac{\pi \hbar c \alpha^{4}}{8 D^{4}}\right)(\text { Volume }) \approx\left(\frac{\pi \hbar c \alpha^{4}}{8 D^{4}}\right)\left(D^{3}\right) \\
& =\frac{\pi \hbar c \alpha^{4}}{8 D} \longrightarrow 0 \quad(D \longrightarrow \infty) .
\end{aligned}
$$

\section{Discussion}

This result that the vacuum energy of the free infinitely large spaces is zero may be interpreted as that the infinite vacuum is a potential resource containing infinitely free virtual particles of negligible frequency which can take higher values of frequency (energy) under the influence of the restrictions made on them by the presence of external boundaries that constrain their infinite freedom; this interpretation seems to be more reasonable than that the vacuum energy for the free infinitely large (or even finite) space has an infinite (irregular) value.

We should mention that the different meaning (interpretation) of energy-time uncertainty relation from the wellknown momentum-position uncertainty principle does not affect what we have calculated here. Indeed, in relation (2), $\Delta \tau$ means a "lifetime" width quantity rather than an uncertainty in time. It is also mentioned that the result of this paper is not in conflict with the response of quantum vacuum to a finite bounded restriction (the Casimir effect). Indeed, although the main "sound" of this paper is that the Casimir energy for free spaces or infinitely large outer spaces in the standard geometries well known in the Casmir effect becomes zero in spite of already accepted infinite (irregular) values, it is possible to find out the expected Casimir force for the wellknown problem of two parallel conducting plates based on the regularization introduced here (see the appendix).

\section{Appendix}

For two plates of distance $d$ from each other, there is a freedom of $x \sim d$ for inner virtual particles and $x \sim D$ $(D \rightarrow \infty)$ for the particles in the two (left and right) outer spaces. As is well known, the Casimir energy corresponding to the famous geometry of two parallel conducting plates is

$$
\begin{aligned}
E_{\text {Casimir }} & =E_{\text {bounded }}-E_{\text {free }} \\
& =\left(E_{\text {left }}+E_{\text {inside }}+E_{\text {right }}\right)-E_{\text {free }} .
\end{aligned}
$$

Considering the resulting relation (6), all three terms $E_{\text {left }}$, $E_{\text {right }}$, and $E_{\text {free }}$ vanish and thus

$$
E_{\text {Casimir }}=E_{\text {inside }} \text {. }
$$

For the case of a scalar field [9], using the well-known energymomentum tensor field

$$
T^{\alpha \beta}=\partial^{\alpha} \varphi \partial^{\beta} \varphi-\frac{1}{2} g^{\alpha \beta} \partial_{\mu} \varphi \partial^{\mu} \varphi
$$

and the following relation between vacuum to vacuum expectation value of the field operators at two space-time points and the time dependent Green function (the propagator)

$$
\left\langle 0\left|T\left\{\varphi(x) \varphi\left(x^{\prime}\right)\right\}\right| 0\right\rangle=-i \hbar c G\left(x, x^{\prime}\right)
$$

and the relation

$$
\begin{aligned}
& \left\langle 0\left|\widehat{T}^{\alpha \beta}\right| 0\right\rangle \\
& =-i \hbar c \lim _{x \rightarrow x^{\prime}}\left(\partial^{\alpha} \partial^{\prime \prime \beta}-\frac{1}{2} g^{\alpha \beta} \partial_{\mu} \partial^{\prime \mu}\right) G\left(x, x^{\prime}\right),
\end{aligned}
$$


by means of

$$
\begin{aligned}
& G\left(x, x^{\prime}\right)_{\text {in }}=\frac{-1}{(2 \pi)^{3}} \int \frac{d \omega}{c} d^{2} k e^{-i \omega\left(t-t^{\prime}\right)} e^{i k_{\perp} \cdot\left(x-x^{\prime}\right)} \\
& \cdot \frac{1}{\lambda \sin \lambda d} \sin \left(\lambda z_{<}\right) \sin \lambda\left(z_{>}-d\right), \\
& \lambda^{2}=\frac{\omega^{2}}{c^{2}}-k^{2}, \\
& \vec{k}_{\perp}=\vec{k}_{x}+\vec{k}_{y} \equiv \vec{k},
\end{aligned}
$$

we arrive at this result that

$$
\begin{aligned}
& \left\langle T^{00}\right\rangle_{\text {in }}=\frac{i \hbar c}{2(2 \pi)^{3}} \int \frac{d \omega}{c} d^{2} k \frac{1}{\lambda \sin \lambda d} \\
& \times\left\{\left(\frac{\omega^{2}}{c^{2}}+k^{2}\right) \sin \lambda z \sin \lambda(z-d)+\lambda^{2} \cos \lambda z\right. \\
& \cdot \cos \lambda(z-d)\} .
\end{aligned}
$$

With the application of complex frequency rotation $(\omega \rightarrow$ $i \omega)$,

$$
\begin{aligned}
& \left\langle T^{00}\right\rangle_{\text {in }}=-\frac{\hbar c}{2(2 \pi)^{3}} \int \frac{d \omega}{c} d^{2} k \frac{1}{\lambda \sinh \lambda d} \\
& \quad \times\left\{\left(-\frac{\omega^{2}}{c^{2}}+k^{2}\right) \sinh \lambda z \sinh \lambda(z-d)+\lambda^{2}\right. \\
& \cdot \cosh \lambda z \cosh \lambda(z-d)\} .
\end{aligned}
$$

After appropriate change of variables and simple integral calculation, the inside energy per unit area is found as

$$
\begin{aligned}
\frac{E_{\text {in }}}{\text { area }} & =\frac{1}{\text { area }} \int\left\langle\widehat{T}^{00}\right\rangle_{\text {in }} d^{3} x=\int_{0}^{d}\left\langle\widehat{T}^{00}\right\rangle_{\text {in }} d z \\
& =-\frac{\hbar c}{6(2 \pi)^{2}} \int_{0}^{\alpha / 2 d} \lambda^{2}((\lambda d) \operatorname{coth} \lambda d+5) d \lambda \\
& =-\frac{\pi^{2} \hbar c}{1440 d^{3}} I(\alpha),
\end{aligned}
$$

in which

$$
I(\alpha)=\frac{3.75}{\pi^{4}} \int_{0}^{\alpha} x^{2}\left(x \frac{e^{x}+1}{e^{x}-1}+10\right) d x, \quad x=2 \lambda d .
$$

As we know, even in the precise measurements (e.g., $[10,11])$, there is no direct experiment confirming the exact numerical coefficient in the already known result $\left(-\pi^{2} \hbar c / 1440 d^{3}\right)$ for the scalar field Casimir pressure because there are experimental difficulties in making two plates parallel at the scales and precisions needed in the modern experiments and unavoidable errors due to working with good real materials rather than perfectly ideal conductors. By the way, one can recover the ideal result by putting $I(\alpha)=1$ which can be achieved by choosing $\alpha \approx 1.874786$ in (A.9); this is an acceptable value for $\alpha$ based on what was explained following relation (1).

\section{Conflict of Interests}

The authors declare that there is no conflict of interests regarding the publication of this paper.

\section{References}

[1] W. Greiner, Quantum Mechanics: Special Chapters, Springer, 1998.

[2] W. Greiner, Quantum Mechanics: Special Chapters, Springer, Berlin, Germany, 1998.

[3] C. Itzykson and J. B. Zuber, Quantum Field Theory, Dover Publications, 2006.

[4] F. Mandl and G. Shaw, Quantum Field Theory, Wiley, 2010.

[5] V. B. Berestetskii, L. P. Pitaevskii, and E. M. Lifshitz, Quantum Electrodynamics, Butterworth-Heinemann, 1982.

[6] M. Guidry, Gauge Field Theories, Wiley-Interscience, 1999.

[7] S. Weinberg, "The cosmological constant problem," Reviews of Modern Physics, vol. 61, no. 1, pp. 1-23, 1989.

[8] H. B. G. Casimir, "On the attraction between two perfectly conducting plates," Proceedings of the Koninklijke Nederlandse Akademie van Wetenschappen, vol. 51, p. 793, 1948.

[9] K. A. Milton, The Casimir Effect: Physical Manifestations of ZeroPoint Energy, World Scientific, Singapore, 2001.

[10] S. K. Lamoreaux, "Demonstration of the casimir force in the 0.6 to $6 \mu \mathrm{m}$ range," Physical Review Letters, vol. 78, article 5, 1997.

[11] U. Mohideen and A. Roy, "Precision measurement of the Casimir force from 0.1 to $0.9 \mu \mathrm{m}$," Physical Review Letters, vol. 81, p. 4549, 1998. 

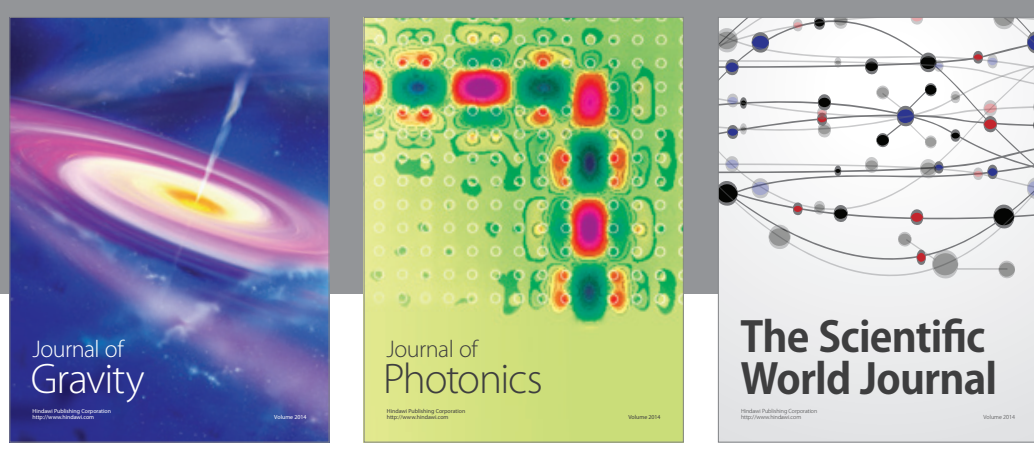

The Scientific World Journal
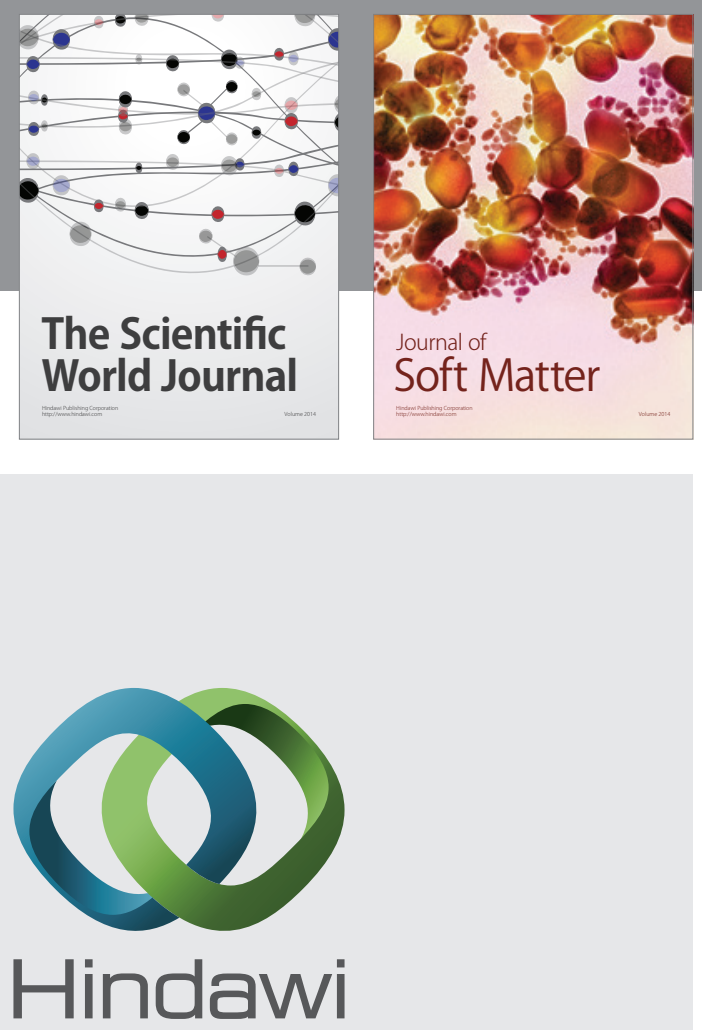

Submit your manuscripts at

http://www.hindawi.com

nternational Journal of

Statistical Mechanics
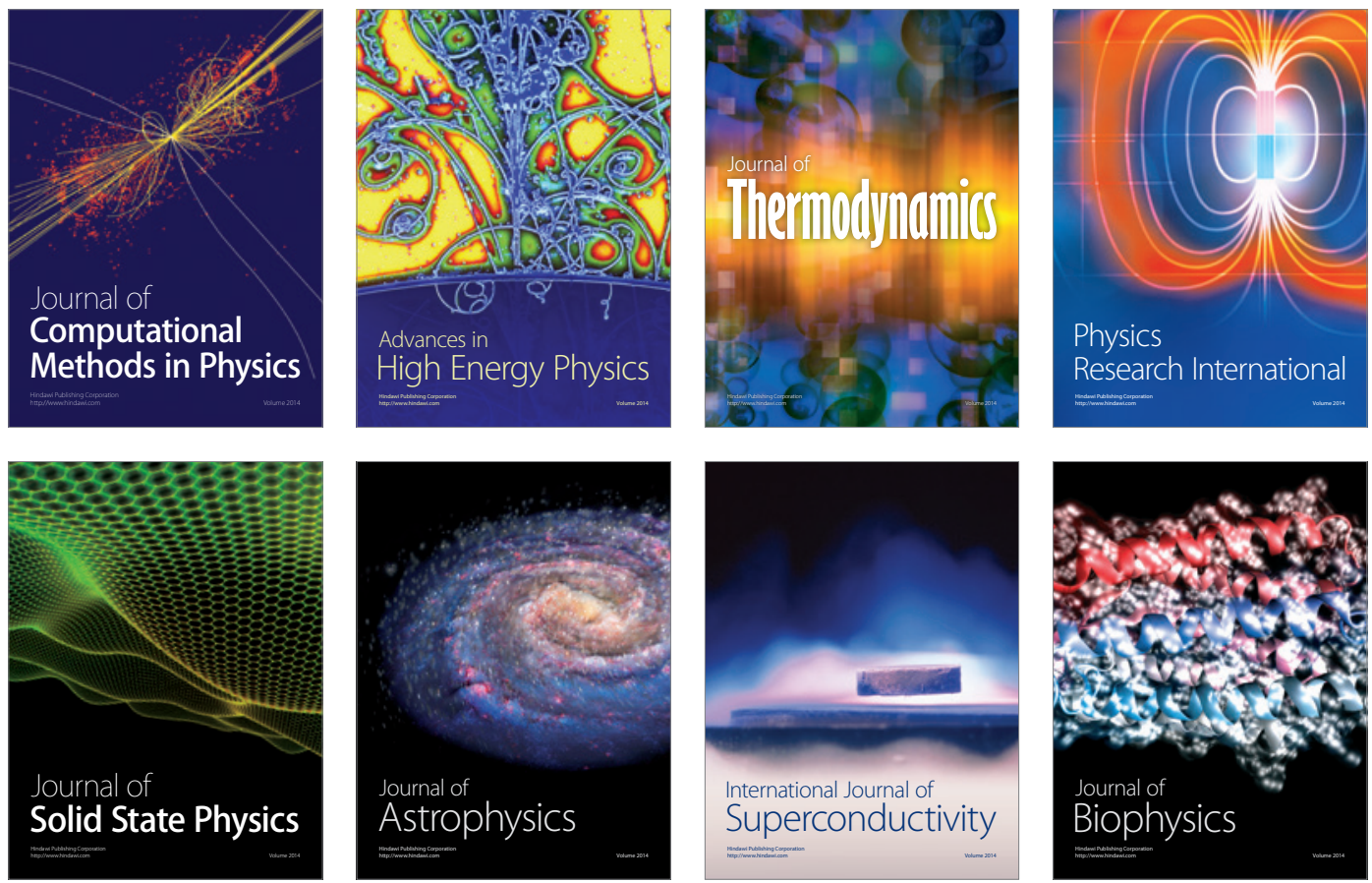
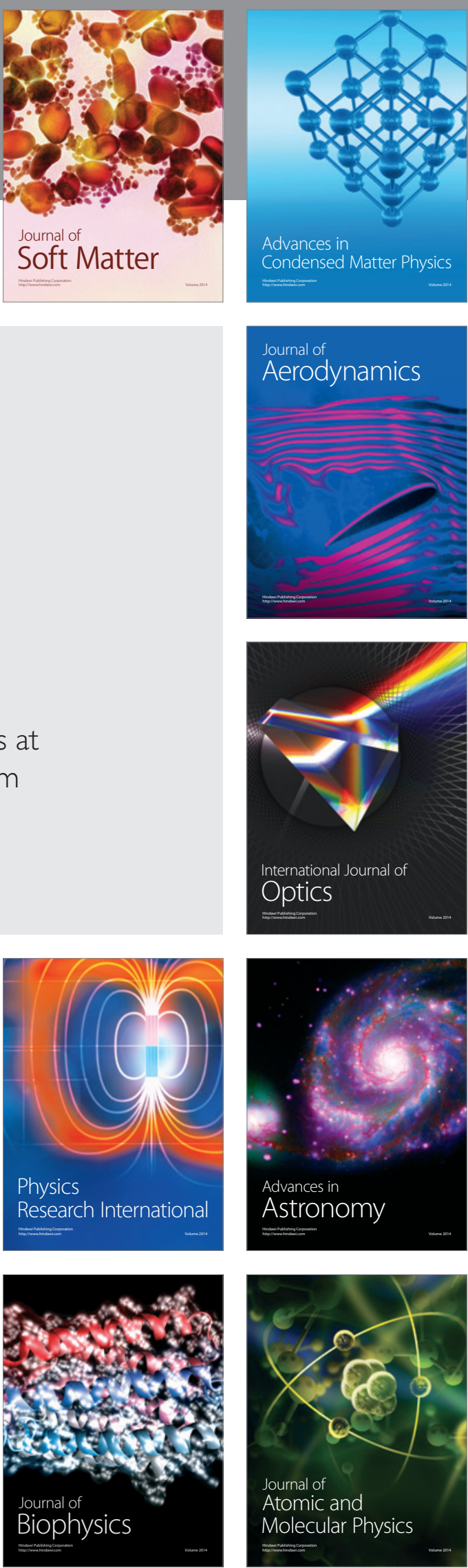